\title{
Vers une justice culturelle. Hommage a Alfredo Gómez-Muller
}

Fecha de entrega: 18 de febrero de 2019

Fecha de evaluación: 7 de septiembre de 2019

Fecha de aprobación: 30 de noviembre de 2019

\author{
Jean-François Petit
}

\section{Résumé}

Philosophe et spécialiste de l'Amérique Latine, Alfredo Gómez-Muller a développé entre France et Colombie une œuvre singulière pour penser la rencontre entre deux mondes. Son « universalisme de la coexistence dialogique » porte singulièrement, à travers divers remaniements théoriques et pratiques, le souci d'une justice culturelle sans cesse affinée. Inspirée par Aristote, Sartre et Marx, elle déjoue tout enfermement dans un supposé inévitable « choc des civilisations ».

Mots-clés : culture, interculturalité, justice culturelle, reconnaissance, rencontre, Amérique Latine.

* Este artículo parte de las investigaciones que ha desarrollado el profesor Petit en torno al pensamiento del filósofo latinoamericano Gómez-Muller. Su intención es resaltar el reencuentro de dos mundos, Colombia y Francia.

Citar como: Petit, J.-F. (2020). Vers une justice culturelle. Hommage a Alfredo Gómez-Muller. Cuadernos de Filosofía Latinoamericana, 41(122), 63-74. Dol: https://doi.org/10.15332/25005375/5528

* Jean-François PETIT, Institut catholique de Paris, UR « religion, culture, société. Diplômé en sciences politiques de I'Institut d'Etudes Politiques de Bordeaux en 1988 (Bordeaux), Diplôme d'Etudes Approfondies en philosophie en 1999 (Université Paris-IV Sorbonne), Capacité doctorale en théologie en 2000 (Institut Catholique de Paris), Docteur en philosophie. Thèse soutenue en 2003 (Institut Catholique de Paris, direction : Ph. Capelle) en cotutelle Université Paris-IV Sorbonne, direction : Alain Renaut.jfpetit@netcourrier.com 


\section{Hacia una justicia cultural: homenaje a Alfredo Gómez-Muller}

\section{Resumen}

El filósofo y especialista en estudios latinoamericanos Alfredo Gómez Muller desarrolló, entre Francia y Colombia, una obra singular para abordar el encuentro entre dos mundos. A través de diversos reajustes teóricos y prácticos, su "universalismo de la coexistencia dialógica" implica, sobre todo, la preocupación por una justicia cultural que se perfecciona constantemente. Inspirada en Aristóteles, Sartre y Marx, aquella impide cualquier forma de aislamiento bajo un, supuestamente inevitable, "choque de civilizaciones".

Palabras clave: cultura, interculturalidad, justicia cultural, reconocimiento, encuentro, América Latina.

\section{Towards a Cultural Justice: An Hommage to Alfredo Gómez-Muller}

\section{Abstact}

The philosopher and Latin American studies specialist Alfredo GómezMuller developed, between France and Colombia, a singular work with which to think the encounter between two worlds. Above all else, his "universalism of dialogic coexistence" entails, through diverse theoretic and practical reworkings, a concern for a cultural justice that is under continuous refinement. Inspired in Aristotle, Sartre, and Marx, such a justice precludes any type of seclusion under a supposedly inevitable "clash of civilizations".

Keywords: culture, interculturality, cultural justice, acknowledgment, encounter, Latin America. 
On ne saurait trop dire le sympathique embarras qui doit légitimement s'emparer de tous ceux qui s'intéresseraient de près à l'œuvre d'Alfredo Gómez-Muller. Déjà, combien ses titres académiques brouillent d'entrée de jeu les pistes! Docteur en philosophie scolastique, habilité par l'université de Strasbourg, licencié en sciences de l'éducation, diplômé des hautes études de l'Amérique Latine de l'Université de Paris III... Ainsi, faut-il le chercher du côté des études hispaniques, c'est-à-dire commencer par la fin, ou par le début en plongeant dans son mémoire de master à l'Institut Catholique de Paris sous la direction de Pierre-Jean Labarrière sur l'amitié dans le chemin de l'anarchie chez Aristote, ou bien du côté de sa thèse sur Heidegger soutenue sous la direction de Jean Greisch (Gómez-Muller, 1986, p. 1). Faut-il le comprendre comme l'artisan de la rencontre entre deux mondes, latino-américain et européen, pour reprendre le titre des quatre leçons publiques de 1992 sur la découverte de l'Amérique (Gómez-Muller, 1993, p. 1) ou le « traquer » à travers ses nombreuses contributions à des dictionnaires comme le Dictionnaire encyclopédique d'éthique chrétienne, des ouvrages collectifs, ou les ouvrages de la collection «Philosophie » de l'Institut Catholique de Paris, qui marquent aussi son goût prononcé du travail en commun? Faut-il être d'abord attentif à ses langues de travail (l'espagnol, le français, l'anglais) ou à certaines de ses responsabilités à l'Institut Catholique de Paris (le Laboratoire de philosophie pratique et d'anthropologie philosophique à partir de 1999 ou le Groupe interdisciplinaire de recherches en éthique) ? Faut-il le chercher du côté des maîtres qu'il a interviewé pour la revue « Concordia » de 1979 à 1999 avec son complice Raul Fornet-Betancourt (Jean-Paul Sartre, Emmanuel Lévinas, Michel Foucault, Karl-Otto Apel, Raimundo Panikkar, Mario Bugne, Julia Kristeva... excusez du peu !) (FornetBetancourt, 2005, p. 377) ou du côté des travaux qu'il a dirigés, en particulier la thèse de Serge Gougbèmon, notre invité ici présent ? (Gougbemon, 2006, pp. 476-489).

On pourrait en conséquence faire d'Alfredo Gómez-Muller un inclassable, tentant, sans succès, d'en trouver une explication du côté de l'anarcho-syndicalisme ou du marxisme, de la Colombie, d'une quête d'un impossible bonheur, d'une critique sans concession d'un universalisme abstrait, d'une réhabilitation des cultures non-occidentales chassées par la colonisation ou maintenue dans une secondarité par la postcolonialité. Mais bien malin qui serait capable d'aller plus loin que ces éléments de finalement cette « anthropologie de la contextualité » qui, pour peu qu'on la comprenne bien, n'a jamais cédé à la facilité ! Et il y a quelques paradoxes à voir les thèses d'Alfredo Gómez-Muller, souvent assez radicales, énoncées avec une apparente délicatesse et un souci pédagogique unanimement salué. 
Cette persévérance dans cette orientation pratique et théorique est sans doute à la mesure des défis, pour ne pas dire des impasses ou des questions encore impensées des champs investigués. Un seul exemple pour me faire comprendre, dans le domaine qui servira ici de fil rouge principal à cette intervention : en 1985, l'Association des théologiens pour l'étude de la morale (ATEM) organise son colloque annuel. Celui-ci s'intitule « Vers une société interculturelle? ». Le Père Valadier, avec la rigueur qu'on lui connait, conclue par une mise en garde qu'il faut citer ici intégralement : « il faut être vigilant : que le pluriculturalisme ne signifie point l'éradication de toutes les traditions, l'uniformisation artificielle, la relativisation de toutes les valeurs, la perte de substance qu'une société qui aurait dilapidé son vouloir vivre en même temps que son identité » (Valadier, 1986, p. 102).

Cependant, en 2011, le point d'exclamation a remplacé le point d'interrogation : «Une société interculturelle est possible ! » titre le numéro des Cahiers de l'Atelier consacré aux premières Assises de l'interculturel, organisées au Centre Sèvres par une vingtaine d'associations et relayé par l'hebdomadaire Témoignage chrétien. Pourtant, sans jouer les Cassandre, Fred Poché, autre intervenant de l'Université catholique de l'Ouest ici présent, y fait quand même remarquer que toutes les formes de reconnaissance, telle que la différence, l'altérité, la diversité, ne sont pas forcément positives et que faire vivre la dimension interculturelle en essayant d'articuler le respect des personnes et le souci du vivre-ensemble reste encore un défi à relever (Poche, 2012, p. 110).

Dès lors, on peut se demander si la tentative d'Alfredo Gómez-Muller de mise en place « d'un universalisme de la coexistence dialogique » (selon ses propres termes) (Gómez-Muller, 1993, p. 377) ne relève pas du " souci d'un passage » (pour reprendre le titre du livre d'hommages adressés à Jean Greisch) (Gómez-Muller, 2004, p. 482) qui n'est pas encore totalement réalisé. On pourrait même faire l'hypothèse qu'Alfredo Gómez-Muller en voit encore plus les difficultés extrêmes. En effet, en 2002, il n’hésite pas à comparer la situation du doyen honoraire de la faculté de philosophie à celle de Descartes, se tenant « un pied dans un pays et l'autre en un autre », trouvant ainsi « sa condition très heureuse, en ce qu'elle est libre » parce que se déployant toujours dans un va et vient entre un « ici » et un « ailleurs » ${ }^{1}$.

1 Lettre de Descartes de 1648 à Elizabeth, cité par A. Gómez-Muller, "Le souci du passage au Nord ". 
L'analyse serait presque de l'ordre de la propre confidence autobiographique heureuse et tranquille si ses derniers ouvrages, en particulier Nihilisme et capitalisme, ne montraient pas l'obstruction grandissante d'un capitalisme contemporain en train de détruire les capacités humaines, de conduire à la mort du sens par la création d'un mode de vie, d'un système de croyances, d'un type de subjectivité individualiste et possessive corrélé à l'appropriation privée et accumulative pensée comme la seule possible et légitime. (Gómez-Muller, 2017, p 50).

En fait, cette critique culturelle du capitalisme, pour peu qu'on la comprenne bien, n'a d'autre ambition que de restituer la légitimité de la diversité des cultures. Bien des figures étudiées par Alfredo Gómez-Muller, à commencer par Bartolomé de las Casas, concourent au projet de renforcement des études culturelles, postcoloniales et subalternes, tout autant que la restauration pratique de la mémoire culturelle et de l'imaginaire politique de peuples ou groupes sociaux marginalisés, dans un accent très « pape François ».

On pourrait dire ici que le nouage symbolique autour de la question de la médiation interculturelle est au service d'une ambition philosophique, à savoir l'établissement d'une justice culturelle. Et tel n'est pas le moindre mérite d'Alfredo Gómez-Muller d'avoir constamment encouragé ses étudiants à investir ces champs de recherche, en les rendant notamment attentifs à la transformation de l'idée des droits de l'homme, en passant des droits libertés aux droits culturels (Bron, 2007, p. 56). Mais là aussi, on se tromperait si l'on dénouait cette question théorique d'un enjeu plus pratique, à savoir l'approfondissement attendu lors de la célébration à l'époque du $60^{\mathrm{e}}$ anniversaire de l'adoption par l'onu de la Déclaration universelle des droits de l'homme, alors que le $70^{\mathrm{e}}$ anniversaire est bien pâle. (Aurenche, 2018).

De son côté, tout en retraçant la généalogie des textes qui établissent ces droits (La convention de l'unEsco pour la protection du patrimoine mondial culturel et naturel en 1972, la Recommandation sur la sauvegarde de la culture traditionnelle et populaire de 1989, la Déclaration universelle sur la diversité culturelle de 2001 et celle de Téhéran sur les droits de l'homme et la diversité culturelle de 2007), Hubert Faës, dans la lignée du " Groupe de Fribourg », né à la suite du colloque « les droits culturels, une catégorie sous-développée des droits de l'homme » organisé par l'Institut Interdisciplinaire d'éthique et de droits de l'homme de Fribourg, aura marqué à juste titre de réelles préventions vis-à-vis d'une perspective qui tendrait peu ou prou à 
« folkloriser, archiver, muséographier les cultures, les figer dans un état d'elle-même » (Faes, 2008, p. 98).

C'est pourquoi on peut considérer la perspective d'Alfredo Gómez-Muller comme un antidote à toute juridisation mal calibrée des droits culturels. Une approche en effet trop abstraite, conjuguant à la hâte égalité et diversité n'est en effet que peu opératoire. Il ne faudrait pas tomber « de Charybde en Scylla » car, de fait, les droits humains dans leur définition courante, sont encore en grande partie une expression de l'individualisme occidental et s'accordent parfois difficilement avec les valeurs culturelles traditionnelles africaines, asiatiques et, dans le cas présent, surtout latino-américaines. Mais si cette assertion cache souvent des stratégies politiques qu'il convient de démasquer, il est vrai que les droits humains et les valeurs culturelles sont partout en tension dans le monde. (Legutke, 2018, p. 218). C’est pourquoi Alfredo Gómez-Muller a constamment cherché des ressources pour favoriser une opérativité de la question interculturelle. Il est indéniable qu'il les a notamment plutôt trouvées du côté principalement de la théorie critique, de la pensée communautarienne et de la philosophie de Sartre.

Cette référence majeure à Sartre est attestée par des biens des travaux qu'on ne peut naturellement ici tous résumer. Cette expérience de l'universel singulier que le directeur des «Temps modernes » a mis en avant à partir d'une herméneutique existentielle, mobilisant à la fois les ressources du récit, de l'anthropologie phénoménologique, de la dialectique et des sciences humaines, explique le geste (Fornet-Betancourt, 2006) philosophique produit par Alfredo Gómez-Muller. En effet, à part Tzvetan Todorov, qui plus que lui a été attentif aux récits de conquête de l'Amérique Latine ? Comment expliquer cet attrait pour la phénoménologie sartrienne si ce n'est pour voir dans la critique du colonialisme de ce dernier non seulement un pourfendeur de l'universalité abstraite mais un précurseur des études postcoloniales. La communication au colloque «Sartre et la culture de l'autre » montre en effet cette orientation : elle porte sur le statut de la culture dans la compréhension sartrienne de la subjectivité, pendant la guerre et dans l'après-guerre, essentiellement dans la Question juive et les Cahiers pour une morale. Mais elle analyse aussi l'expression éthique de la compréhension subjectivement située de l'universalisme abstrait de la pratique libérale individualiste en restant orientée par le projet d'une culture qui se recréé sans cesse dans les formes de la praxis. Dès lors sont bien posés les jalons d'un universel concret conçu en tant que "création interculturelle de l'éthique comme noyau transculturel des cultures" (Gómez-Muller, 2017, p. 123-124). 
Cette perspective de recherche n'aura pas échappé à Raul Fornet-Betancourt, autre ténor de la philosophie interculturelle. Non seulement elle fait justice à Sartre en tant que philosophe, en dépassant l'horizon de compréhension eurocentriste qui marque de fait la pratique philosophique de son temps, en ouvrant ainsi des voies pour le dialogue interculturel mais, en même temps, elle fait justice à son engagement pour les droits culturels de l'autre (Fornet-Betancourt, 2005, p. 146). De même qu'il y a un «Bergson postcolonial », il n'est pas inadéquat de dire qu'il y a un « Sartre postcolonial » (Diagne, 2011, p. 27) qui aura été marqué par un souci constant à l'égard des questions relatives à la diversité culturelle, que Raul Fornet Betancourt, de façon transversale, fait aller des textes sur le " problème noir » aux Etats-Unis jusqu'aux entretiens avec Benny Levy. (Sartre, 199, p. 87).

Cette compréhension d'un nécessaire approfondissement des conditions de rencontres interculturelles est étroitement corrélée à la mobilisation d'une compréhension latino-américaine de l'existence, faisant du droit à la vie une dimension pratique de l'agir éthico-pratique plus que la réaffirmation d'un principe formel intangible. Alfredo Gómez-Muller donne ici la priorité à des hommes et des femmes concrets aux raisonnements éthiques, qui plus est, se situent, en position concurrentielle. Bien souvent, les éthiciens font l'impasse sur les registres de précompréhensions morales et politiques qui fondent les prises de position. Ainsi, ils omettent de préciser que nos conceptions éthiques contemporaines se forgent dans la culture morale et politique de la modernité libérale du XvII ${ }^{\mathrm{e}}$ siècle. De ce fait, celles-ci ne sont pas si neutres qu'elles veulent bien le dire.

C'est pourquoi la recherche d'une justice interculturelle ne peut pas se dissocier de l'étude de la constitution de la mémoire éthique. A ce stade, à une compréhension individualiste de l'éthique comme finalité sans fin, Alfredo Gómez-Muller préfère et trouve plus juste d'interroger la constitution de l'émergence du sens, la possibilité de fins pratiques dans les déterminations sociales et l'intersubjectivité historique qui les a constitués. C'est pourquoi, à plusieurs endroits, son travail est attentif aux traditions pratiques des communautés historiques. Il ne peut donc y avoir de justice interculturelle sans mémoire interculturelle. Or, celle-ci fait souvent défaut, comme dans le cas de la politique républicaine française concernant l'esclavage. De cette mémoire expurgée ne peut émerger qu'une conception déficiente de la prise en charge réflexive de l'exigence humaine de sens (Gruzinski, 1988, p. 99) . A bien des égards, ce positionnement se rapproche de l'analyse historique et systématique du concept 
de vertu par Alasdair MacIntyre - Alfredo Gómez-Muller étant un bon connaisseur du débat entre " libéraux » et « communautariens » (Gómez-Muller, 2001, p. 675)contestant en cela un individualisme libéral négligeant les conditions culturelles mais aussi sociales et économiques qui déterminent les conceptions éthiques. C'est dans un processus plus général de refus d'une uniformisation du symbolique que cette critique prend place. L'expurgation de la mémoire des traditions pratiques conduit à la détermination de finalités sociales exclusives ou d'acquiescement à des situations de coexistence culturelle, parfois bancales, à un moment donné.

On peut donc dire que la recherche d'une justice interculturelle est tout entière une protestation contre ce que Charles Taylor a pu qualifier «d'aplatissement » ou de « rétrécissement » de la vie des sociétés. Alfredo Gómez-Muller se trouve ici en droit de mettre en question une démocratie qui, "portée par le souci 'd'égalité' apparait comme un 'énorme balai' qui fait disparaitre les mémoires éthico-symboliques » (Gómez-Muller, 1999, p. 32). On pourrait croire en une filiation tocquevillienne. En fait, l'auteur se situe plus dans le sillage du second Sartre en cherchant à montrer comment la théorie dialectique, l'herméneutique et la phénoménologie, ont contribué au développement d'une anthropologie comprenant la subjectivité d'abord comme intersubjectivité. On aurait pu aussi rapprocher cette perspective de la conception de la mémoire de Ricœur, à cette époque en cours d'élaboration (Ricœur, 2000) à ceci près qu'Alfredo Gómez-Muller me semble avoir été avant tout soucieux d'un dépassement du conflit entre mémoire et liberté. La culture, de ce point de vue, ne se situe pas forcément du côté, de l'histoire, de la mémoire, de la tradition, si on veut bien la considérer comme vivante, déploiement social historique, toujours en quête de réélaboration de ses contenus. Dès lors, on mesure ici la lourde tâche de ceux qui voudraient participer à la re-constitution intersubjective d'une mémoire interculturelle. Cette orientation reviendrait à accepter « l'acte de perpétuelle re-création intersubjective, aux niveaux narratif, théorique et pratique, du sens, des fins et des valeurs de l'agir et de la (co) existence » (Gómez-Muller, 1999, p. 34).

Cette communication n'entendant pas faire nombre avec celle du Père Adrián García sur la partie colombienne, je me contenterai de souligner l'attention constante d'Alfredo Gómez-Muller au cri des pauvres d'Amérique Latine, qui "exprime l'inconditionné universel qui précède toute argumentation et fonde la possibilité de toute argumentation» (Gómez- Muller, 1988, p. 488). Il faudrait mesurer ici l'ampleur de la référence lévinasienne plus que sartrienne dans cette approche cherchant à faire 
droit aussi bien au son, au regard, au geste, à l'image, à l'écriture, qui sont toujours adressés à quelqu'un. On pourrait compléter ici Lévinas en se demandant si le visage d'autrui n'est pas le commencement même de la philosophie interculturelle. Comment ne pas voir que la relation interhumaine est au fondement même de la rencontre interculturelle et que la responsabilité éthique qui s'exerce vis-à-vis d'autrui, de l'autre homme, du prochain, inclut nécessairement ses origines et ses orientations culturelles, y compris quand il appartient à un peuple. La constitution d'une anthropologie de l'universalité des cultures, où aucune d'elle ne sera retranchée et qui ne se résume pas à sa version occidentale, pour ne pas dire européenne, passe bien par ce niveau premier d'appréhension. Les pièges des théories évolutionnistes, culturalistes, ethnocentriques, essentialistes de la culture peuvent sans doute être partiellement déjoués si nous restons "à hauteur de visage ». Des " chocs culturels " pourraient être évités à condition d'adopter sans naïveté une posture de reconnaissance, amicale et attentionnée des autres cultures, sans naïveté, c'est-à-dire notamment aussi sans ethnocentrisme inversé.

Ce n'est donc pas un hasard qu'Alfredo Gómez-Muller ait porté une attention soutenue aux problématiques de la reconnaissance. Il n'est pas surprenant que le volume collectif dédié à cette question de Laboratoire d'anthropologie et de philosophie pratique (LANPRAT) s'ouvre précisément sur cette interrogation : «Avec le développement de l'exigence de justice culturelle, dans le monde contemporain, la notion de reconnaissance est en train d'acquérir une importance nouvelle dans la vie des personnes, des groupes et des sociétés » (Gómez- Muller, 2009, p. 12). Certes, une meilleure prise en compte de catégorie de reconnaissance du point de vue des conditions d'auto-réalisation de la subjectivité, explicitant ainsi sa fonction critique dans le cadre de la philosophie sociale y est pour beaucoup (Honneth, 2000). Par contre, le couplage avec la question interculturelle est le fait des auteurs de ce collectif. Alors qu'Hubert Faës y traite de la dimension anthropologique de la reconnaissance ; dialectique pour Raul Fornet Betancourt et de cette " voie moyenne » entre diversité culturelle et universalisme éthique, Alfred Gómez-Muller y prend en charge une problématique alliant « reconnaissance, culture et idéologie». Ce dernier texte, assez synthétique, me semble comme résumer les axes majeurs de cette recherche étalée sur plus d'une trentaine d'années : la revendication de justice culturelle a d'abord un fond anthropologique, car celle-ci permet la reconnaissance et la promotion de l'identité de la personne. Ce fut le cas depuis les travaux pionniers d'Edward Sapir depuis 1916 jusqu’à la recherche plus contemporaine de Charles Taylor, Will Kymlicka et Michael 
Walzer d'une citoyenneté multiculturelle ${ }^{2}$. Mais l'effort ne doit pas être moindre du côté éthique, la justice culturelle contribuant à une " vie bonne » individuelle et collective, l'évacuation de cette dimension participant au maintien d'une domination instituée. Et en dernier ressort, c'est au sein de l'ensemble de la société que cette question de la justice culturelle prend sens, la culture apparaissant à maints égards comme le rempart symbolique où chacun s'abrite pour défendre la signification de son existence. Dès lors, il n'y a qu'un pas à franchir pour affirmer que la crise de la culture est une crise de la symbolisation. Clifford Geertz estime à cet égard que la modernité achève la transformation de la culture en idéologie ou en simple valeur d'échange, récapitulation accumulative d'un perpétuel présent. C'est pourquoi, dans bien des cas, la revendication de justice culturelle compte en elle une exigence de justice sociale.

$\mathrm{Au}$ final, on ne saurait sous-estimer la radicalité des positions obtenues ici par la transformation d'un regard quelque peu irénique sur l'interculturalité. On le voit bien, il ne s'agit pas seulement d'un ton en philosophie ou de la recherche de critères argumentatifs adaptés à la situation latino-américaine. Sans faire un inventaire complet des espaces d'interculturalité ni des disciplines requises, la perspective d'Alfredo Gómez Muller, qui se situe très efficacement dans l'esprit «Laudato Si », revient aussi à répondre concrètement à l'interpellation : «qu'as-tu fait de ton frère?».

\section{Bibliographie}

Aurenche, G. (2018). Droits humains, noublions pas notre idéal commun. Paris : Ed. Temps présent.

Bron, A. (2007). Transformations de l'idée des droits de l'homme: des droits libertés aux droits culturels, Mémoire de Master sous la direction d'Alfredo Gómez Muller. Paris : Institut catholique de Paris/Université de Poitiers.

Diagne, B. (2011). Bergson postcolonial. CNRs Editions.

Faes, H. (2008). Droits de l'homme et droits culturels. Transversalités, 108, 85-99.

2 Cf. E. Sapir, Anthropologie, Minuit, 1967, t 1. Culture et personnalité ; C. Taylor, Multiculturalisme, différence et démocratie, Aubier, 1994 ; W. Kymlicka, La citoyenneté multiculturelle, La Découverte, 2001. 
Fornet-betancourt, R. (2006). L'humanisme solidaire de Sartre : anticipation de l'universalité et de la philosophie interculturelles. Dans A. Gómez-Muller, Sartre et la culture de l'autre, L'Harmattan (pp. 99-124). Paris : Editions L'Harmattan.

Fornet-Betancourt, R., \& Gómez-Muller, A. (2005). Léthique en dialogue. Paris : Pleins feux.

Fornet-Betancourt, R., \& Gómez-Muller, A. (2005). Léthique en dialogue. Paris : Ed. Pleins feux.

Gómez-Muller, A. (1986). Laliénation comme inauthenticité chez Martin Heidegger, thèse de doctorat canonique en philosophie sous la direction de Jean Greisch. Paris : Institut Catholique de París.

Gómez-Muller, A. (1988). Le droit à la vie, valeur politique. Etudes, 369, 484.

Gómez-Muller, A. (1993). Coexistence des sens et sens de la coexistence. Dans : Comprendre et interpréter, le paradigme herméneutique de la raison. Philosophie(15), 377.

Gómez-Muller, A. (1993). Penser la rencontre entre deux mondes 1993. Paris : PUf.

Gómez-Muller, A. (1999). Mémoire, liberté, pluralisme. Revue d’éthique et de théologie morale, 210, 21-36.

Gómez-Muller, A. (2001). Les communautariens et la critique de l'individualisme libéral : Alasdair Mac Intyre, Charles Taylor, Michael Walzer. En A. Caille, C. Lazzeri, \& M. Senellart, Histoire raisonnée de la philosophie morale et politique (pp. 672-681). Paris : La Découverte.

Gómez-Muller, A. (2004). Le souci du passage au Nord. Dans P. Capelle, G. Hebert, \& M.-D. Popelard, Le souci du passage, Mélanges offerts à Jean Greisch (pp. 476489). Paris : Cerf.

Gómez-Muller, A. (2006). Sartre et la culture de l’autre. Paris : L'Harmattan.

Gómez-Muller, A. (2009) (Dir.). La reconnaissance : réponse à quels problèmes? Paris : L'Harmattan.

Gómez-Muller, A. (2017). Nihilisme et capitalisme. Paris : Kimé. 
Gougbemon, S. (2006). Problématique et éthique dans la pensée de Sartre : vers la constitution d'une éthique concrète, thèse de doctorat de philosophie. Paris : Institut Catholique de Paris.

Gruzinski, S. (1988). La colonisation de l'imaginaire. Sociétés indigènes et occidentalisation dans le Mexique espagnol XVI-XvIIIe siècle. Paris : Gallimard.

Honneth, H. (2006). La lutte pour la reconnaissance. La Découverte : La société du mépris.

Legutke, D. (2018). Droits humains et traditions culturelles. Spiritus, 231, 213-224.

Poche, F. (2012). Interculturalité, un équilibre à trouver entre le commun et la différence. Cahiers de l'atelier, 535, 110.

Ricoeur, P. (2000). La mémoire, l'histoire, l'oubli. Paris : Gallimard.

Sartre, J.-P. (16 juin et 30 juillet de 1945). Retour des Etats Unis. Ce que j’ai appris du problème noir. Le Figaro littéraire.

Sartre, J.-P., \& Levy, B. (1991). Lespoir maintenant. Paris : Verdier.

Todorov, T. (1982-1983). La conquête de l’Amérique; Récits aztèques de conquête. Seuil : Nous et les autres.

ValadieR, P. (avril de 1986). Vers une société interculturelle? Le supplément, Revue d'éthique et de théologie morale, 156, 102. 